\title{
Erratum to: A Crosstalk between the Biorhythms and Gatekeepers of Longevity: Dual Role of Glycogen Synthase Kinase-3
}

\author{
Gregory A. Shilovsky ${ }^{1,2,3, a *}$, Tatyana S. Putyatina ${ }^{2}$, Galina V. Morgunova ${ }^{2}$, \\ Alexander V. Seliverstov ${ }^{3}$, Vasily V. Ashapkin ${ }^{1}$, Elena V. Sorokina ${ }^{2}$, \\ Alexander V. Markov ${ }^{2}$, and Vladimir P. Skulachev ${ }^{1}$ \\ ${ }^{1}$ Belozersky Institute of Physico-Chemical Biology, Lomonosov Moscow State University, 119991 Moscow, Russia \\ ${ }^{2}$ Faculty of Biology, Lomonosov Moscow State University, 119234 Moscow, Russia \\ ${ }^{3}$ Institute for Information Transmission Problems, Russian Academy of Sciences, 127051 Moscow, Russia \\ ${ }^{a} e$-mail: gregory_sh@list.ru; grgerontol@gmail.com
}

Received July 14, 2020

Revised January 1, 2021

Accepted January 1, 2021

DOI: $10.1134 / \mathrm{S} 0006297921050096$

The original article can be found online at https://doi.org/10.1134/S0006297921040052

On p. 444 before "Ethics declarations" section should read:

Funding. This research was supported by the Russian Foundation for Basic Research (project no. 18-29-13037). 